\title{
Origins of the Martian aurora observed by Spectroscopy for Investigation of Characteristics of the Atmosphere of Mars (SPICAM) on board Mars Express
}

\author{
F. Leblanc, ${ }^{1}$ O. Witasse, ${ }^{2}$ J. Winningham, ${ }^{3}$ D. Brain, ${ }^{4}$ J. Lilensten, ${ }^{5}$ P.-L. Blelly, ${ }^{6}$ \\ R. A. Frahm, ${ }^{3}$ J. S. Halekas, ${ }^{4}$ and J. L. Bertaux ${ }^{1}$ \\ Received 4 April 2006; revised 26 May 2006; accepted 9 June 2006; published 22 September 2006.
}

[1] On the 11 August 2004, the UV spectrograph Spectroscopy for Investigation of Characteristics of the Atmosphere of Mars (SPICAM) on board Mars Express made the first observation of auroral-type emission on the Martian nightside. In this paper, we describe the results of a new analysis of the observed emission owing to a better calibration of SPICAM UV channel and the use of all spectral information obtained during this observation. Several possibilities for the origin of this emission are discussed. We discussed, in particular, the possible exact geometry of the observation and the possible origins of the Martian aurorae. The emissions measured by SPICAM ultraviolet spectrometer have most probably been produced by electrons with an energy distribution peaking at few tens of $\mathrm{eV}$ rather than by electron distributions peaking above $100 \mathrm{eV}$.

Citation: Leblanc, F., O. Witasse, J. Winningham, D. Brain, J. Lilensten, P.-L. Blelly, R. A. Frahm, J. S. Halekas, and J. L. Bertaux (2006), Origins of the Martian aurora observed by Spectroscopy for Investigation of Characteristics of the Atmosphere of Mars (SPICAM) on board Mars Express, J. Geophys. Res., 111, A09313, doi:10.1029/2006JA011763.

\section{Introduction}

[2] During orbit 716 of the Mars Express mission, Spectroscopy for Investigation of Characteristics of the Atmosphere of Mars (SPICAM) UV spectrometer clearly identified an auroral-type emission [Bertaux et al., 2005a]. A significant increase of the measured emission was observed for seven seconds on the Martian nightside. This additional emission was significantly different than the already well-identified nightglow emission [Bertaux et al., $2005 \mathrm{~b}]$. It was composed, in particular, of the $\mathrm{CO}\left(\mathrm{a}^{3} \Pi-\right.$ $\left.\mathrm{X}_{\sim}^{1} \Sigma^{+}\right)$Cameron band system emission and of the $\mathrm{CO}_{2}^{+}$ $\left(\tilde{\mathrm{B}}^{2} \Sigma_{\mathrm{u}}^{+}-\tilde{\mathrm{X}}^{2} \Pi_{\mathrm{g}}\right)$ doublet emission which are typically observed in the Martian dayglow [Leblanc et al., 2006]. The auroral-type emission was estimated to be located at $\sim 178^{\circ}$ east longitude, $\sim-50^{\circ}$ latitude, and $129 \pm 13 \mathrm{~km}$ altitude. The very high correlation between this position and the presence of the strongest crustal magnetic field structures identified at Mars [Acuňa et al., 2001] strongly suggests that

\footnotetext{
${ }^{1}$ Service d'Aéronomie du Centre National de la Recherche Scientifique/ Institut Pierre-Simon Laplace, Verrières-le-Buisson, France.

${ }^{2}$ Research and Scientific Support Department of European Space Agency-European Space Research and Tecnology Centre, Noordwijk, Netherlands.

${ }^{3}$ Southwest Research Institute, San Antonio, Texas, USA.

${ }^{4}$ Space Sciences Laboratory, University of California, Berkeley, California, USA.

${ }_{5}^{5}$ Laboratoire de Planétologie de Grenoble, Grenoble, France.

${ }^{6}$ Laboratoire de Physique et Chimie de l'Environnement, Orléans, France.

Copyright 2006 by the American Geophysical Union. 0148-0227/06/2006JA011763
}

these structures play a role in guiding the incident particles responsible for these emissions.

[3] Since the report of this first observation, auroral-like peaked electron distributions have been identified in the Mars Global Surveyor data set [Brain et al., 2006a] as well as by Mars Express [Lundin et al., 2006a, 2006b]. This type of electron distribution, largely observed in association with the Earth's aurora, displays peak energies at Mars ranging from a few hundred eV up to $4 \mathrm{keV}$ and is observed in good correlation with regions close to the Martian crustal magnetic fields [Brain et al., 2006a; Lundin et al., 2006b]. The peaked electron distributions have been explained as being produced by acceleration by parallel electric fields along magnetic field lines [Lundin et al., 2006a, 2006b]. Brain et al. [2006a] also suggested the occurrence of the encounter of a solar energetic particles event with Mars at the moment of SPICAM ultraviolet spectrograph (UVS) observation.

[4] In this paper, we present a new analysis of the SPICAM UVS observation (section 1), then we discuss the shape of the auroral emission, in particular, based on what is known of the morphology of the crustal magnetic field (section 2). In section 3, we introduce several potential electron distributions typical of the Martian environment in order to discern the origins of SPICAM UVS observations. These different electron distributions are used as the upper boundary for a model of the Martian airglow. Such a model describes the energy degradation of downward electrons through the Martian atmosphere and is coupled to a one-dimensional (1-D) model of the transport of the ionized atmospheric particles. This model calculates the OI emission line at $297 \mathrm{~nm}$, the $\mathrm{CO}\left(\mathrm{a}^{3} \Pi-\mathrm{X}^{1} \Sigma^{+}\right)$Cameron band system emission, and the $\mathrm{CO}_{2}^{+}\left(\tilde{\mathrm{B}}^{2} \Sigma_{\mathrm{u}}^{+}-\tilde{\mathrm{X}}^{2} \Pi_{\mathrm{g}}\right)$ doublet emission that is generated by the electron flux (section 4$)$. We conclude 
Table 1. Orbit 716 (11 August 2004) Position of the Spacecraft and Mars Nearest Point During the Auroral Observation ${ }^{\mathrm{a}}$

\begin{tabular}{lccccccc}
\hline Time, UT & SPICAM Time & S/C Altitude, $\mathrm{km}$ & S/C Longitude & S/C Latitude & MNP Altitude, $\mathrm{km}$ & MNP Longitude & MNP Latitude \\
\hline $0601: 21$ & 533 & 267 & 166.96 & -53.08 & 15.4 & 198.7 & -46.25 \\
$0601: 28$ & 540 & 266.3 & 167.01 & -53.55 & 15.0 & 199.06 & -46.69 \\
\hline
\end{tabular}

${ }^{\mathrm{a}}$ Mars nearest point (MNP). The coordinates of the MNP are given at the center of the field of view of SPICAM UVS.

in section 5 on the needed characteristics of the electron distribution in order to reproduce the SPICAM UVS observation of auroral emission.

\section{Measured Auroral Emissions}

[5] The SPICAM UV spectrograph (UVS) is part of the SPICAM light instrument (also composed of an IR spectrograph covering $1.2-4.8 \mu \mathrm{m}$ which is dedicated to solar occultations and nadir viewing). Information on the SPICAM UVS instrument is given by Bertaux et al. [2000, 2006]. A typical observation of the Martian airglow by SPICAM UVS provides five different spectra, corresponding to five adjacent regions on the CCD. Each of these regions, which we have named spatial bin, corresponds to different adjacent fields of view. In the case of an airglow observation, a slit is mechanically placed at the focal plane of the first mirror in order to achieve a spectral resolution adapted to airglow measurements. This slit has two parts; one narrow part with $1.5 \mathrm{~nm}$ spectral resolution and one larger part with $6 \mathrm{~nm}$ resolution (the later having $\sim 8$ times better sensitivity than the former). For most of the airglow measurements [Leblanc et al., 2006] and in particular in the case of the auroral observation we will discuss hereafter [Bertaux et al., 2005a], two of the spatial bins correspond to the narrow part of the slit and two to the large part of the slit. The fifth spatial bin corresponds to both the large and narrow parts and is difficult to calibrate accurately.

[6] The first observation of a Martian aurora was reported by Bertaux et al. [2005a]. We provide in Table 1 the main positions and times of this observation. The spectrum, as measured through the narrow part of the slit, has been published in Figure 3 of Bertaux et al. [2005a]. Because the emission brightness was not very intense, part of the Cameron bands appeared to be missing in their figure. It was this uncertainty which caused us to reevaluate the spectra, and make further comparisons between aurora and dayglow using the wide part of the slit. In Figure 1, we display the sum of the two spectra obtained through the large part of the slit (Figure 1b) as well as a typical spectrum of the Martian dayglow seen through the large part of the slit (Figure 1a). When using this wider part of the slit, the larger sensitivity of SPICAM UVS allows much better resolution of the entire emission associated with this aurora. As a consequence, we can conclude that the main features of the Martian dayglow, namely, the $\mathrm{CO}\left(\mathrm{A}^{1} \Pi-\mathrm{X}^{1} \Sigma^{+}\right) 4 \mathrm{P}$ bands, the $\mathrm{CO}\left(\mathrm{a}^{3} \Pi-\right.$ $\left.\mathrm{X}^{1} \Sigma^{+}\right)$Cameron band system emission, and the $\mathrm{CO}_{2}^{+}\left(\mathrm{B}^{2} \Sigma_{\mathrm{u}}^{+}-\right.$ $\mathrm{X}^{2} \Pi_{\mathrm{g}}$ ) doublet emission are clearly seen in the spectra of the aurora. The Lyman alpha emission is not apparent in Figure $1 \mathrm{~b}$ because this emission has been suppressed when subtracting the nightglow emission (see Bertaux et al. [2005a] for further explanations).
[7] The calibration of SPICAM UVS is accomplished by use of star observations [Bertaux et al., 2006] which are regularly made during the Mars Express (MEX) mission. The calibration used by Bertaux et al. [2005a] has recently been greatly improved thanks to the observation of stars with parameters of operation of SPICAM UVS close to the parameters of operation used for airglow observations (with a slit and a high gain on the microchannel plates). Such observations allowed us to determine a new effective area [Bertaux et al., 2006] significantly different than the one displayed in Figure 3a of Bertaux et al. [2005a]. This new effective area has a similar dependency in wavelength but is between 1.6 to 1.8 times smaller than the one used by Bertaux et al. [2005a]. We also improved our method of integrating the total signal corresponding to each emission. In order to fit each emission line, we used a model of the instrument point spread function which consisted of the product of the convolution of an ideally thin emission and of the spectral response function [Bertaux et al., 2005a]. However, it appears that this method leads to inaccurate estimates of the integrated emission brightness. As explained by Leblanc et al. [2006], we now favor a direct integration of the emission line. A second improvement has been performed in the method used to subtract the background emission which is particularly important when estimating the total Cameron band emission intensity. Table 2 provides the new estimates of the intensity for the features which are clearly identified in the auroral spectrum. Table 2 provides the published intensities of Bertaux et al. [2005a].

[8] The emission intensity of the Cameron band system deduced from CCD spatial bins 0 and 1 is slightly greater than deduced from CCD spatial bins 3 and 4. An explanation for this difference is that spatial bins 0 and 1 are more centered on the CCD than spatial bins 3 and 4. Therefore these bins are less sensitive to the aberration of the lens which can explain the 30\% difference from spatial bin 1 to spatial bin 4 (E. Dimarillis, personal communication, 2005). Another explanation comes from the fact that part of the light coming from the large slit can diffuse through the input window and increase the signal measured on the CCD corresponding to spatial bins 0 and 1 [Bertaux et al., 2006]. As a consequence, within the uncertainty, a conservative estimate for the measured emission intensities is for the $\mathrm{CO}\left(\mathrm{a}^{3} \Pi-\right.$ $\mathrm{X}^{1} \Sigma^{+}$) Cameron band emission $2_{-0.4}^{+0.5} \mathrm{kR}$. In the case of the $\mathrm{CO}_{2}^{+}\left(\tilde{\mathrm{B}}^{2} \Sigma_{\mathrm{u}}^{+}-\tilde{\mathrm{X}}^{2} \Pi_{\mathrm{g}}\right)$ ultraviolet doublet near $289 \mathrm{~nm}$, the best estimate for the intensity is $200_{-100}^{+90} \mathrm{R}$. In the case of the $\mathrm{O}\left({ }^{1} \mathrm{~S}-{ }^{3} \mathrm{P}\right) 297.2 \mathrm{~nm}$ emission, only spatial bin 1 has a clear feature that can be associated with this emission with intensity of $89 \pm 91 \mathrm{R}$ and is therefore only marginally identified. It may contribute to the integrated intensity displayed in Table 2 and may be associated with the $\mathrm{CO}_{2}^{+}\left(\tilde{\mathrm{B}}^{2} \Sigma_{\mathrm{u}}^{+}-\tilde{\mathrm{X}}^{2} \Pi_{\mathrm{g}}\right)$ ultraviolet doublet near $289 \mathrm{~nm}$ in 


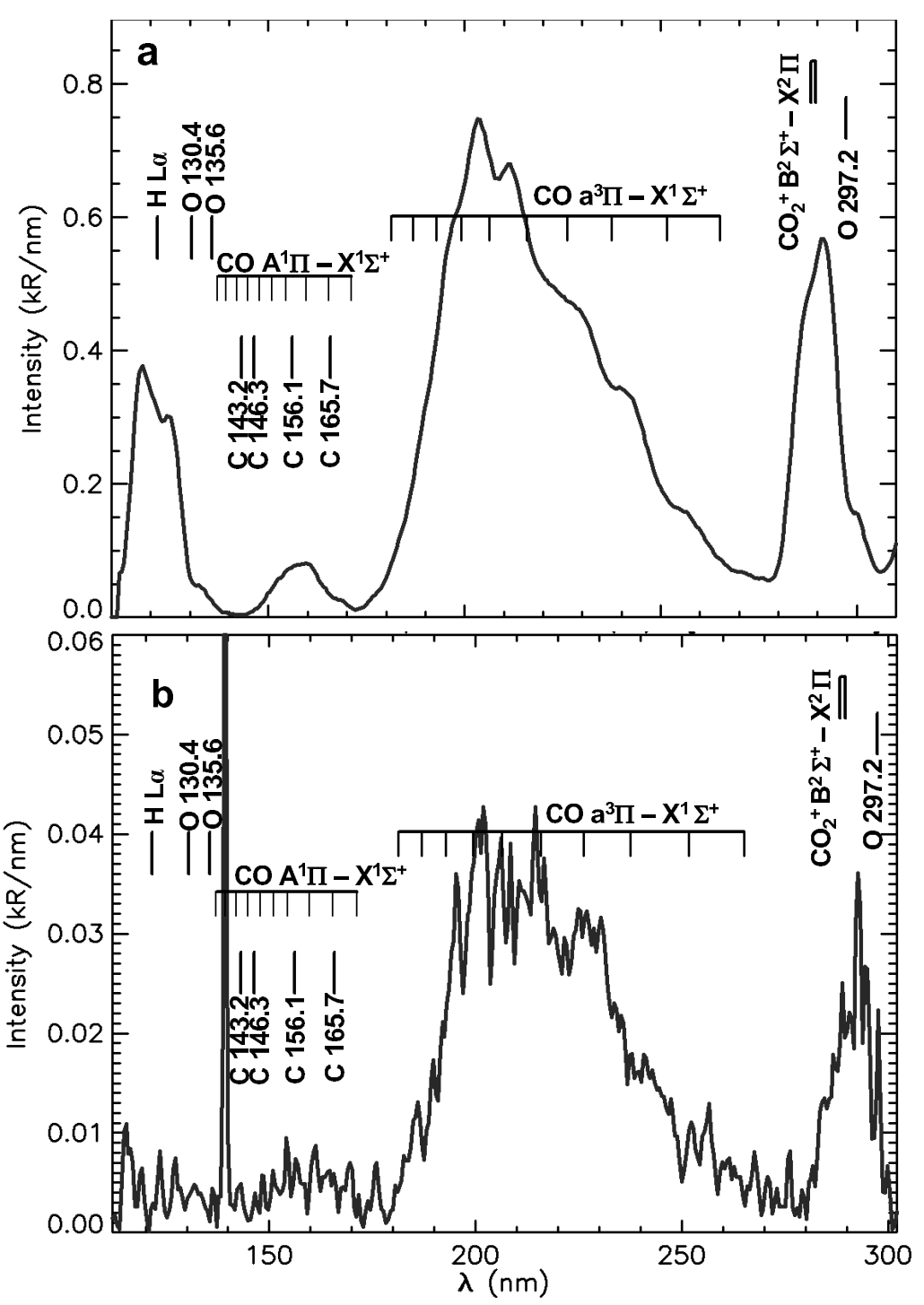

Figure 1. (a) Spectrum of the dayglow emission as seen by the large part of the slit. This spectrum consists of a 1125 spectra average obtained on the Martian dayside between altitudes of 140 and $150 \mathrm{~km}$, SZA between $14^{\circ}$ and $85^{\circ}$, and Ls between $100^{\circ}$ and $171^{\circ}$. (b) Average spectrum of the emissions as seen by the part of the CCD corresponding to the large part of the slit (spatial bins 3 and 4). The nightglow emission has been subtracted from this spectrum following the method described by Bertaux et al. [2005a]. The peak at $140 \mathrm{~nm}$ is due to a cosmic particle which impacts the CCD (seen only on spatial bin 4).

spatial bins 3 and 4 corresponding to the large part of the slit (Figure 1).

\section{Configuration of the Observation}

[9] During Mars Express orbit 716, SPICAM UVS was pointed tangentially toward the limb with a field of view oriented perpendicular to the spacecraft velocity. Mars Express was at an altitude of $266 \mathrm{~km}$, whereas the tangent point of the field of view (Mars nearest point of the field of view) was at an altitude of 15 and $1365 \mathrm{~km}$ away from Mars Express. The observations of the aurora by SPICAM UVS indicated that the region of emission was $30 \mathrm{~km}$ wide perpendicular to the SPICAM UVS field of view. This result was deduced from the duration of the enhancement of the signal and the velocity of the satellite during this observation. Indeed, this duration corresponds to the time spent by the SPICAM UVS field of view inside the region of emission. We excluded the possibility of a transient event because all 5 spatial bins saw the same duration event, but with a slight delay associated with the difference in their fields of view. In order to quantify the flux of electrons needed to reproduce this observation we considered as a first approximation that the region should have also been circular and extended $30 \mathrm{~km}$ along the direction of the field of view of SPICAM UVS [Bertaux et al., 2005a]. However, because the emission region has been estimated as being roughly at a distance of $450 \pm 50 \mathrm{~km}$ from the spacecraft, a much longer path length of SPICAM field of view through the auroral region cannot be excluded. The maximum possible length can be 
Table 2. Average Intensity of the Emissions Over the Entire Period of the Auroral Observations ${ }^{\text {a }}$

\begin{tabular}{cccc}
\hline & & $\begin{array}{c}\text { Integrated } \\
\text { Emission, kR }\end{array}$ & $\begin{array}{c}\text { Integrated Emission } \\
\text { From Bertaux et al. } \\
{[2005 \mathrm{a}], \mathrm{kR}}\end{array}$ \\
\hline 0 & $\mathrm{CO}\left(\mathrm{a}^{3} \Pi-\mathrm{X}^{1} \Sigma^{+}\right)$ & $2.2 \pm 0.15$ & $0.694 \pm 0.05$ \\
1 & & $2.5 \pm 0.16$ & \\
3 & & $1.9 \pm 0.04$ & \\
4 & & $1.6 \pm 0.04$ & \\
0 & $\mathrm{CO}_{2}{ }^{+}\left(\tilde{\mathrm{B}}^{2} \Sigma_{\mathrm{u}}^{+}-\tilde{\mathrm{X}}^{2} \Pi_{\mathrm{g}}\right)$ & $0.09 \pm 0.06$ & $0.07 \pm 0.042$ \\
1 & & $0.24 \pm 0.09$ & \\
3 & & $0.28 \pm 0.04$ & \\
4 & & $0.21 \pm 0.03$ & \\
\hline
\end{tabular}

${ }^{\mathrm{a}}$ For seven individual spectra. These intensities are deduced from the spectra measured by each individual spatial bins. Spatial bins 0 and 1 correspond to the part of the CCD which sees the narrow part of the slit whereas, spatial bins 3 and 4 correspond to the wide part of the slit.

estimated as being equal to $\sim 900 \mathrm{~km}$. Such a length corresponds to the distance between Mars Express and the closest point of the field of view of SPICAM which is at an altitude around $60 \mathrm{~km}$. Indeed below such an altitude, the $\mathrm{CO}_{2}$ absorption considerably reduces any atmospheric emission above $175 \mathrm{~nm}$. Therefore any emission occurring in the field of view of SPICAM UVS at a distance larger than $900 \mathrm{~km}$ to MEX should be significantly attenuated before reaching SPICAM UVS.

[10] Electron pitch angle distributions have been recorded by the Mars Global Surveyor (MGS) magnetometer/electron reflectometer every $2-8 \mathrm{~s}$ during the spacecraft mapping orbit phase at $\sim 400 \mathrm{~km}$ altitude. Pitch angle distributions recorded in a single instrument energy channel $(115 \mathrm{eV}$, a channel typically uncontaminated by photoelectrons) have been classified according to their shape. Figure 2 shows the probability, as a function of geographic location on the Martian night side, of observing a statistically greater flux of electrons returning from the planet than moving toward the planet [Brain et al., 2006b]. These distributions are interpreted as having single-sided loss cones, indicative of open magnetic field lines (connected at one end to the draped IMF and at the other to the Martian exobase or crust). Also shown in Figure 2 is the respective position of Mars Express, the estimated position of the auroral emission, and the Mars nearest point of the SPICAM UVS field of view. As shown in Figure 2, the region where electrons from high altitude may have precipitated into the Martian atmosphere (along magnetic open field lines) could have been very elongated along the SPICAM field of view.

[11] Figure 2 suggests therefore that contrary to the assumption made by Bertaux et al. [2005a], the auroral region could have been much more elongated parallel than perpendicular to the field of view of SPICAM UVS. As discussed by Bertaux et al. [2005a], a $450 \mathrm{~km}$ path length along the auroral region (rather than $30 \mathrm{~km}$ ) would imply that the electron flux required to reproduce the observation could be smaller than inferred by Bertaux et al. [2005a], that is more than 10 times the intensity of the typical magnetosheath flux reported by MGS [Mitchell et al., 2001].

\section{Electron Energy Distributions Measured in the Martian Environment}

[12] A second interesting point suggested by Figure 2 is that during the SPICAM UVS observation, Mars Express could have crossed the same region of open magnetic field lines associated with the auroral emission. As a consequence, during the time of the observation by SPICAM, the Analyzer of Space Plasmas and Energetic Atoms (ASPERA-3) on board Mars Express and in particular the electron spectrometer (ELS) [Barabash et al., 2004] may have measured a signature of the electrons precipitation into the Martian



Figure 2. Map of the probability (expressed in percentage) to be in an open field line region at $400 \mathrm{~km}$ in altitude on the Martian nightside as calculated from the electron reflectometer on board Mars Global Surveyor during its mapping phase orbit. Also plotted are the positions of Mars Express at the time of the auroral observation by SPICAM UVS, the estimated position of the auroral region and the position of Mars nearest point of the SPICAM UVS field of view. The white line represents the field of view of SPICAM UVS. 




Figure 3. Electron distributions measured in the Martian environment. Solid line without symbols, MGS/ER measurement of a flux electron spike [Mitchell et al., 2001, Figure 8]. Solid line with diamonds, MGS/ER measurement of the magnetosheath electron distribution [Mitchell et al., 2001, Figure 8]. Solid line with pluses, MEX/ASPERA-3 measurement of the electron downward flux during the SPICAM UVS auroral observation. A correction for the $-4 \mathrm{~V}$ spacecraft potential has been applied to the measurement. Solid line with crosses, MGS/ER measurement of an electron auroral-type distribution [Brain et al., 2006b].

atmosphere associated with the auroral emission. At the time of the observation by SPICAM UVS, ASPERA-3 did indeed observe an electron spike-type event. Electron spikes have been observed by MGS/ER in correlation with the presence of a strong radial component of the Martian magnetic crustal field [Acuòa et al., 2001; Mitchell et al., 2001]. In Figure 3, the solid line without symbols displays the typical spike electron energy distribution observed by MGS/ER. However, in the case of ASPERA-3 this electron spike has a significantly different energy distribution, with two peaks of the flux at 12.3 and $17 \mathrm{eV}$ (Figure 3, solid line with plus symbols). Such peaks have been identified as being produced by the ionization on the dayside of $\mathrm{CO}_{2}$ molecules by the strong HeII $304 \AA$ solar line with theoretical peaks at 21-24 and $27 \mathrm{eV}$ [Mantas and Hanson, 1979]. Their observed shift toward lower energy is attributed to spacecraft potential and energy cascading due to inelastic scattering during the electron travel [Frahm et al., 2006; Liehmon et al., 2006]. In the case of this measurement the spacecraft potential has been estimated as being around $-4 \mathrm{~V}$ from nearby time period but this estimate suffers from a significant uncertainty due to the particular magnetic and local time region which is crossed by MEX at that time. In Figure 3 we also plot two other types of electron distribution that have been measured in the Martian environment, in particular, the typical magnetosheath electron energy distribution measured by MGS/ER [Mitchell et al., 2001] that was used by Bertaux et al. [2005a] and the auroral-type electron distribution recently reported by Brain et al. [2006a].

[13] At the time of the auroral measurement by SPICAM UVS, MEX was at a solar zenith angle of $117.6^{\circ}$ and a local time of approximately 2000 LT, whereas the Mars nearest point of the SPICAM UVS field of view was at 2110 LT and the auroral region was at local time near 2030 LT. At $2000 \mathrm{LT}$ and an altitude of $266 \mathrm{~km}$, MEX was therefore in the penumbra of Mars. The photoelectrons observed by ASPERA-3 may therefore have been formed locally but this solution is very unlikely due to the intensity of the observed flux. Most probably, these electrons have been transported from the dayside. Actually, ASPERA-3 has reported the observations of photoelectron-type distributions deep in the magnetotail of Mars suggesting that such transport occurs very often in the Martian environment [Frahm et al., 2006]. The reconnection of the interplanetary magnetic field lines draped around Mars with the strong crustal fields at late evening could have allowed dayside photoelectrons to be transported to MEX. It remains difficult, however, to explain how such photoelectrons could have been transported to a region where the Martian auroral emission has been observed. In section 5, we use all electron distributions displayed in Figure 3 to infer the properties of the energy distributions responsible for the observed emissions.

\section{Analysis of the Measured Auroral Emissions}

[14] In order to simulate the emissions that may be produced by the different electron distributions displayed in Figure 3, we have used Transcar, a 1-D model based on a coupling between kinetic and fluid codes. The kinetic part uses a stationary Boltzmann approach and describes the energetic electron flux. The fluid part is an eight moment up to the heat flow time-dependent model that solves the transport equations for different charged species. This code has been extensively and successfully used to describe the Earth's ionosphere [Blelly et al., 1996; Lilensten and Blelly, 2002]. It has been adapted to the Martian atmosphere by using the Viking atmospheric conditions [e.g., Witasse et al., 2002; Morel et al., 2004]. The neutral species introduced in Transcar are for the Martian atmosphere $\mathrm{N}_{2}, \mathrm{O}_{2}, \mathrm{O}, \mathrm{H}, \mathrm{CO}_{2}$ and $\mathrm{CO}$. The variations of their density profiles for Mars are 
Table 3. Emission Intensities Calculated by Transcar for Two Different Assumptions on the Size of the Aurora Regions Along SPICAM UVS Field of View

\begin{tabular}{|c|c|c|c|c|}
\hline Electron Distributions & 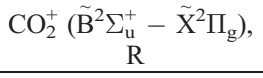 & $\frac{\mathrm{O}\left({ }^{1} \mathrm{~S}-{ }^{3} \mathrm{P}\right)}{\mathrm{R}}$ & $\begin{array}{c}\mathrm{CO}\left(\mathrm{a}^{3} \Pi-\mathrm{X}^{1} \Sigma^{+}\right) \\
\mathrm{R}\end{array}$ & 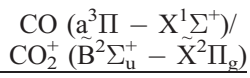 \\
\hline \multicolumn{5}{|c|}{ Length of the FOV Through the Aurora Extended up to $900 \mathrm{~km}$ From MEX } \\
\hline MGS Aurora & 83 & 57 & 219 & 2.7 \\
\hline MGS Spike & 1.6 & 0.1 & 5.2 & 3.2 \\
\hline MGS Sheat & 45 & 3.9 & 190 & 4.2 \\
\hline MEX/ASPERA-3 (orbit 716) & 168 & 12 & 643 & 3.8 \\
\hline \multicolumn{5}{|c|}{ Length of the FOV Through the Aurora Extended From $435 \mathrm{~km}$ to $465 \mathrm{~km}$ From MEX } \\
\hline MGS Aurora & 4.6 & 0.3 & 13 & 2.8 \\
\hline MGS Spike & 0.1 & 0.01 & 0.3 & 2.6 \\
\hline MGS Sheat & 2. & 0.1 & 5.3 & 2.6 \\
\hline MEX/ASPERA-3 (orbit 716) & 14 & 0.8 & 36 & 2.6 \\
\hline
\end{tabular}

given in Figure 2 of Fox and Dalgarno [1979b]. The Martian atmospheric temperature increases from $120^{\circ} \mathrm{K}$ up to $200^{\circ} \mathrm{K}$ from 100 to $150 \mathrm{~km}$ [Nier and McElroy, 1977]. Above $150 \mathrm{~km}$, the temperature is constant and equal to $200^{\circ} \mathrm{K}$. Cross sections for ionization and excitation by electrons of the main Martian components as well as reaction rates are given by Fox and Dalgarno [1979a, 1979b] and Tian and Vidal [1998]. In the Martian nightside atmosphere, the $\mathrm{CO}\left(\mathrm{a}^{3} \Pi-\mathrm{X}^{1} \Sigma^{+}\right)$Cameron band system emission is essentially produced by electron impact dissociation of the $\mathrm{CO}_{2}$ atmospheric molecules leading to the $\mathrm{CO}\left(\mathrm{a}^{3} \Pi\right)$ state. $\mathrm{CO}_{2}^{+}\left(\tilde{\mathrm{B}}^{2} \Sigma_{\mathrm{u}}^{+}-\tilde{\mathrm{X}}^{2} \Pi_{\mathrm{g}}\right)$ doublet emission is produced by ionization of the $\mathrm{CO}_{2}$ atmospheric molecules leading to the $\mathrm{CO}_{2}^{+}\left(\widetilde{\mathrm{B}}^{2} \Sigma_{\mathrm{u}}^{+}\right)$state. For these two reactions, we used cross sections peaking at $0.6 \times 10^{-16} \mathrm{~cm}^{2}$ for the production of the $\mathrm{CO}_{2}^{+}\left(\widetilde{\mathrm{B}}^{2} \Sigma_{\mathrm{u}}^{+}\right)$ state [Fox and Dalgarno, 1979b] and at $0.7 \times 10^{-16} \mathrm{~cm}^{2}$ for the production of the CO $\left(\mathrm{a}^{3} \Pi\right)$ state [Conway, 1981]. Above the magnetic anomalies, the transport of the charged particles is assumed to be along the magnetic field lines which are vertical and constant in intensity between 100 and $500 \mathrm{~km}$ in altitude. The electrons are injected at $500 \mathrm{~km}$ with an initial isotropic pitch angle distribution.

[15] We have tested Transcar by comparing our results with the only published work calculating the emissions associated with magnetospheric electrons impacting the Martian nightside atmosphere [Haider et al., 1992]. Good agreement was found between the emission rates for the Cameron band system emission calculated by Haider et al. [1992] and our calculation using the same electron distribution (electron fluxes coming from either the sheet or the tail as determined by Phobos measurements [Verigin et al., 1991]), ionization and excitation cross sections for $\mathrm{CO}_{2}$ by electron impacts, and the neutral atmosphere model used by Haider et al. [1992]. In the case of the $\mathrm{CO}_{2}^{+}\left(\tilde{\mathrm{B}}^{2} \Sigma_{\mathrm{u}}^{+}-\tilde{\mathrm{X}}^{2} \Pi_{\mathrm{g}}\right)$ ultraviolet doublet near $289 \mathrm{~nm}$, Transcar predicts a peak of 3 photons $\mathrm{cm}^{-3} \mathrm{~s}^{-1}$ at $162 \mathrm{~km}$ and 10 photons $\mathrm{cm}^{-3} \mathrm{~s}^{-1}$ at $136 \mathrm{~km}$ for the Phobos magnetotail and magnetosheeth electron distributions which can be compared with Haider et al. calculations. These emission rates are roughly proportional by a factor of 5 to the $\mathrm{CO}_{2}^{+}$impact ionization rates from Haider et al. [1992] (they calculated an ionization rate by electronic impact equal to 25 and 60 ions $\mathrm{cm}^{-3} \mathrm{~s}^{-1}$ at the ionospheric peak for magnetotail and magnetosheath distri- butions, respectively). These rates are in good agreement with the Transcar results of 15 and 50 ions $\mathrm{cm}^{-3} \mathrm{~s}^{-1}$ at roughly the same altitude. In the case of the Cameron band system emission, we compared Transcar emission rates to the ones provided by Haider et al. [1992] and found a similar agreement. As a conclusion, within a factor 2, Transcar provides estimates of the emission rates of the $\mathrm{CO}\left(\mathrm{a}^{3} \Pi-\mathrm{X}^{1} \Sigma^{+}\right)$Cameron band system and the $\mathrm{CO}_{2}^{+}\left(\widetilde{\mathrm{B}}^{2} \Sigma_{\mathrm{u}}^{+}-\tilde{\mathrm{X}}^{2} \Pi_{\mathrm{g}}\right)$ doublet in good agreement with Haider et al. [1992].

[16] We used the different electron distributions displayed in Figure 3 as an upper boundary condition for Transcar. Using the field of view configuration of SPICAM UVS, we calculated the Transcar intensities of the two identified emissions during the auroral event. Two different lengths of the aurora region were considered: a spherical $30 \mathrm{~km}$ wide region, as by Bertaux et al. [2005a], and a $900 \mathrm{~km}$ elongated aurora region parallel to the field of view and centered on the estimated position of the aurora. The results of these calculations are given Table 3. As discussed in section 1, during this auroral event, SPICAM UVS clearly identified only two atmospheric emissions: namely, the $\mathrm{CO}\left(\mathrm{a}^{3} \Pi-\mathrm{X}^{1} \Sigma^{+}\right)$ Cameron band emission with an intensity between 1600 and $2500 \mathrm{R}$ and the $\mathrm{CO}_{2}^{+}\left(\widetilde{\mathrm{B}}^{2} \Sigma_{\mathrm{u}}^{+}-\widetilde{\mathrm{X}}^{2} \Pi_{\mathrm{g}}\right)$ ultraviolet doublet near $289 \mathrm{~nm}$ with an intensity between 90 and 290 R. For both shapes of the auroral region, the intensities calculated by Transcar using the electron magnetosheath (MGS Sheath), MGS spike, MGS auroral-type electron distribution or ASPERA-3 ELS distributions are significantly less intense than that measured by SPICAM UVS. Moreover, the ratio between the $\mathrm{CO}\left(\mathrm{a}^{3} \Pi-\right.$ $\left.\mathrm{X}^{1} \Sigma^{+}\right)$Cameron band emission and the $\mathrm{CO}_{2}^{+}\left(\tilde{\mathrm{B}}^{2} \Sigma_{\mathrm{u}}^{+}-\tilde{\mathrm{X}}^{2} \Pi_{\mathrm{g}}\right)$ ultraviolet doublet near $289 \mathrm{~nm}$ identified by SPICAM UVS is significantly different than that calculated for any of these electron distributions. The value of the ratio of the two measured emissions is between 6 and 28 with a most probable value around 10 (and even larger if the $\mathrm{CO}_{2}^{+}$ $\left(\tilde{\mathrm{B}}^{2} \Sigma_{\mathrm{u}}^{+}-\tilde{\mathrm{X}}^{2} \Pi_{\mathrm{g}}\right)$ ultraviolet doublet near $289 \mathrm{~nm}$ emission observed during this aurora-type event was contaminated by the $\mathrm{CO}_{2}^{+}\left(\tilde{\mathrm{A}}^{2} \Pi_{\mathrm{u}}^{+}-\tilde{\mathrm{X}}^{2} \Pi_{\mathrm{g}}\right)$ band system $)$. As displayed in Table 3 , the highest value of this ratio is obtained for the electron magnetosheath or ASPERA-3-type distributions, but remains significantly smaller than the measured ratio (by a factor 2.5). The auroral-type electron distribution provides 


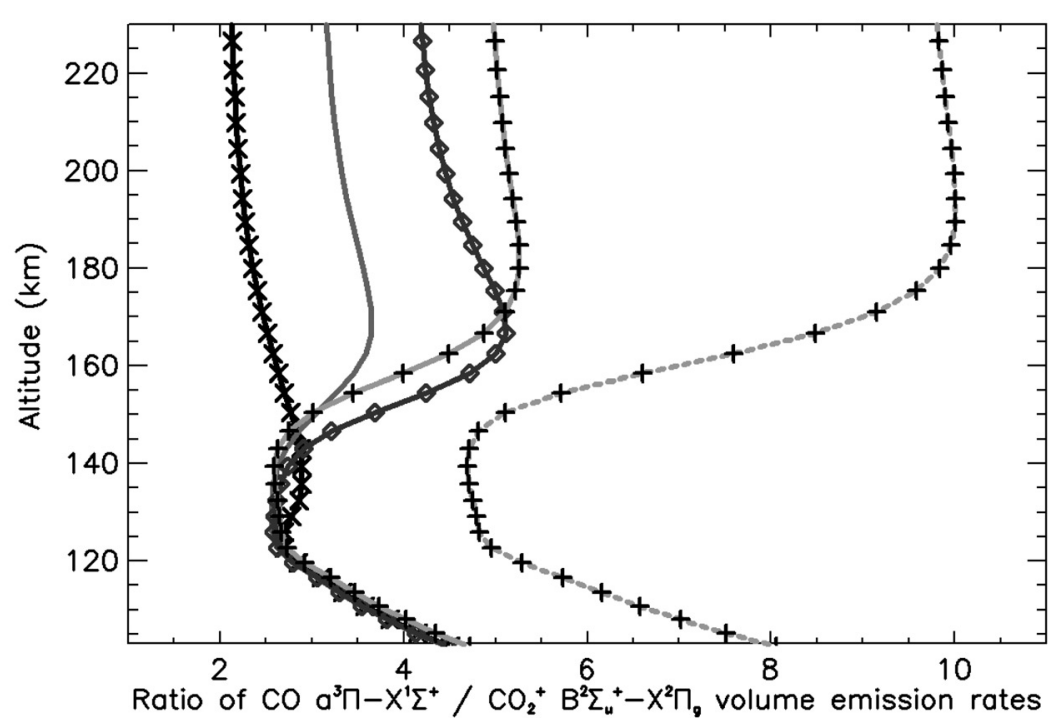

Figure 4. Ratio versus altitude between the volume emission rates of the $\mathrm{CO}\left(\mathrm{a}^{3} \Pi-\mathrm{X}^{1} \Sigma^{+}\right)$Cameron band system and of the $\mathrm{CO}_{2}^{+}\left(\widetilde{\mathrm{B}}^{2} \Sigma_{\mathrm{u}}^{+}-\tilde{\mathrm{X}}^{2} \Pi_{\mathrm{g}}\right)$ doublet. Same legend as for Figure 3. Dashed line with pluses, MEX/ASPERA-3 measurement (same as solid line with crosses) but using a 2 times larger cross section of dissociation excitation of the CO Cameron band system.

the smallest value of the ratio regardless of the geometry of the auroral region (smaller than the measured ratio by a factor $\sim 4$ ).

[17] The largest ratio is obtained when the simulated emission is integrated over the largest altitude range. As displayed in Figure 4, the altitude range of the observed auroral region (with estimated altitude of $129 \pm 13 \mathrm{~km}$ ) is typically where the ratio of these two emissions is the smallest. This ratio is the largest above $150 \mathrm{~km}$ in altitude for all of the electron distributions except the auroral-type distribution. The cross section of the main process leading to the formation of the excited states has been described by Fox and Dalgarno [1979a] and discussed by Fox [1992]. The cross section of the excitation of the $\mathrm{CO}_{2}^{+}\left(\widetilde{\mathrm{B}}^{2} \Sigma_{\mathrm{u}}^{+}\right)$ state peaks around $60 \mathrm{eV}$ whereas the cross section for the excitation of the $\mathrm{CO}\left(\mathrm{a}^{3} \Pi\right)$ state peaks around $20 \mathrm{eV}$. On the dayside, the photoelectron population peaks significantly below $60 \mathrm{eV}$ which induces a Cameron band system emission much larger than the $\mathrm{CO}_{2}^{+}\left(\tilde{\mathrm{B}}^{2} \Sigma_{\mathrm{u}}^{+}-\right.$ $\mathrm{X}^{2} \Pi_{\mathrm{g}}$ ) ultraviolet doublet. The ratio of the Cameron band system emission and of the $\mathrm{CO}_{2}^{+}\left(\tilde{\mathrm{B}}^{2} \Sigma_{\mathrm{u}}^{+}-\tilde{\mathrm{X}}^{2} \Pi_{\mathrm{g}}\right)$ ultraviolet doublet emission typically observed by SPICAM UVS varies from 4 to 8 with increasing solar zenith angle [Leblanc et al., 2006]. An auroral-type electron distribution peaking above $100 \mathrm{eV}$, such as the solid line with crosses in Figure 3, produces a ratio of these two emissions larger than 3 only when the electron population has lost a significant part of its initial energy through the Martian atmosphere, that is, below the ionospheric peak (between 125 and $140 \mathrm{~km}$ for Viking neutral atmospheric conditions).

[18] We have tested the sensitivity of these results with respect to the neutral atmosphere by performing the same calculation with a Mariner 6 and 7 type atmosphere (that is with an exospheric temperature $\sim 300^{\circ} \mathrm{K}$ ). In that case, the altitude of the ionospheric peak changed by a few kilometers which is not enough to significantly change the results displayed in Table 3. A differently shaped auroral region (as an example extended between MEX and the estimated position of the aurora region) does not change the ratios displayed in Table 3. Tests of different initial pitch angle electron distributions (from fully isotropic to fully forward oriented distributions) again did not significantly change the ratios displayed in Table 3. Another source of uncertainty that we have considered in this work is related to the cross sections. There is a large uncertainty, by up to a factor 2 in the absolute value of the cross section leading to the formation of the $\mathrm{CO}$ $\left(\mathrm{a}^{3} \Pi\right)$ state [Fox, 1992]. Indeed, Erdman and Zipf [1983] have suggested that the absolute cross section for the production of $\mathrm{CO}\left(\mathrm{a}^{3} \Pi\right)$ state could peak at $2.4 \times 10^{-16} \mathrm{~cm}^{2}$ rather than at $0.7 \times 10^{-16} \mathrm{~cm}^{2}$ as suggested by Conway [1981]. However, as discussed by Fox [1992] such a value for the cross section would lead to much larger emission intensities in the Martian atmosphere than observed. The dashed line with plus symbols illustrates the change that can be associated with a cross section for the excitation of the $\mathrm{CO}$ $\left(a^{3} \Pi\right)$ state that is two times larger $\left(1.4 \times 10^{-16} \mathrm{~cm}^{2}\right)$. In such a case, the ratio of the two calculated emissions may reach a value close to that observed by SPICAM UVS if the field of view of the instrument crosses the auroral region above $150 \mathrm{~km}$ in altitude.

[19] As discussed in section 4, a major unknown in the measurement made by ASPERA-3 is the spacecraft potential. We estimated a potential of $-4 \mathrm{~V}$ from ASPERA-3 measurements made a few seconds before the SPICAM UVS observation However, a $-4 \mathrm{~V}$ potential implies a significant energy loss during the electron travel with respect to the theoretical $21-24 \mathrm{eV}$ and $27 \mathrm{eV}$ peaks of the Martian photoelectron. Indeed, the uncorrected measurements from the spacecraft potential have peaks at 8.3 and $12 \mathrm{eV}$. This suggests a maximum possible spacecraft potential up to $-14 \mathrm{~V}$. We used this value to correct the electron distribution measured by ASPERA-3 and Transcar with a cross section of $0.7 \times 10^{-16} \mathrm{~cm}^{2}$ for the 
production of the $\mathrm{CO}\left(\mathrm{a}^{3} \Pi\right)$ state and an assumed $900 \mathrm{~km}$ long auroral region. We found $2 \mathrm{kR}$ and $300 \mathrm{R}$ for the Cameron band system emission and for the $\mathrm{CO}_{2}^{+}\left(\widetilde{\mathrm{B}}^{2} \Sigma_{\mathrm{u}}^{+}-\right.$ $\left.\widetilde{\mathrm{X}}^{2} \Pi_{\mathrm{g}}\right)$ ultraviolet doublet emission, respectively. We calculate $1.15 \mathrm{kR}$ and $224 \mathrm{R}$ for a spacecraft potential of $-10 \mathrm{~V}$. This result is in surprisingly good agreement with the SPICAM observation. It suggests therefore that SPICAM UVS has observed atmospheric emissions produced by electrons with an energy distribution similar to the typical dayside photoelectron energy distribution, i.e., airglow due to transported photoelectrons.

[20] The typical auroral-type electron distributions reported by Brain et al. [2006a] should produce a nadir viewing intensity equal to $\sim 60$ and $\sim 23 \mathrm{R}$ for the Cameron band system emission and for the $\mathrm{CO}_{2}^{+}\left(\tilde{\mathrm{B}}^{2} \Sigma_{\mathrm{u}}^{+}-\tilde{\mathrm{X}}^{2} \Pi_{\mathrm{g}}\right)$ ultraviolet doublet emission, respectively. With a limb viewing (with a MNP of the field of view at $110 \mathrm{~km}$ in altitude), such an emission would have been equal to $790 \mathrm{R}$ and $294 \mathrm{R}$ for the Cameron band system intensity and for the $\mathrm{CO}_{2}^{+}\left(\tilde{\mathrm{B}}^{2} \Sigma_{\mathrm{u}}^{+}-\widetilde{\mathrm{X}}^{2} \Pi_{\mathrm{g}}\right)$ ultraviolet doublet emission, respectively. These latter intensities are within the sensitivity of SPICAM UVS [Leblanc et al., 2006] and therefore should be easily detectable on the nightside by SPICAM UVS with a limb viewing. Lundin et al. [2006a, 2006b] published electron spectra recorded at higher altitudes that they associated to auroral-type acceleration with significant higher intensities than the ones reported by Brain et al. [2006a]. Such electron distributions would have produced emissions with intensity between $\sim 0.5$ up to $\sim 5 \mathrm{kR}$ and $\sim 0.1$ and $1 \mathrm{kR}$ for the Cameron band system emission and for the $\mathrm{CO}_{2}^{+}\left(\widetilde{\mathrm{B}}^{2} \Sigma_{\mathrm{u}}^{+}-\tilde{\mathrm{X}}^{2} \Pi_{\mathrm{g}}\right)$ ultraviolet doublet emission, respectively, when observed with a nadir viewing (between 10 and $100 \mathrm{kR}$ and between 3 and $30 \mathrm{kR}$ with a limb viewing geometry). These intensities are of the order of the dayside observed emissions [Leblanc et al., 2006] and therefore should also be easily observed on the nightside with either nadir or limb viewing.

[21] Finally, there are two other sources that can significantly influence the distribution of the electrons in term of energy. The first one is linked to proton precipitation. Haider et al. [2002] compared the effects of solar EUV and electron-proton precipitation on the ion production (and not on the energy distribution of the secondary electrons). They show that the proton influence cannot be ruled out even in dayside conditions. Up to now, there is no solver able to compute the energy distribution of secondary electrons due to primary protons in the ionosphere of Mars. It is therefore not possible to compare this effect to the measurements. The second source of uncertainty is the magnetic field strength itself. In our simulation, we suppose that the field is constant at altitude above $100 \mathrm{~km}$, as we do on Earth. However, the magnetic source on Earth is located in the core of the planet, so that in spite of a $1 / \mathrm{r}^{3}$ decrease, there is not much difference between the lower and the upper ionospheres. It could be quite different at Mars, where the magnetic source is probably located close to the surface [Acuòa et al., 2001]. From the conservation of the first adiabatic invariant, a change in the magnetic field intensity results in a change in the pitch angle and therefore in the perpendicular velocity of the incoming primary particles. This affects, in turn, the energy distribution of the secondaries and should also change the altitude of the brightest emission. Here again, it has not been possible to check this mechanism through our modeling.

\section{Conclusions}

[22] The MEX auroral-type emissions have been reanalyzed after the calibration of the SPICAM UVS was improved. We also discussed the potential origins of these emissions considering different electron energy distributions: namely, the Mars Global Surveyor magnetosheath and spike electron distributions [Mitchell et al., 2001], Mars Global Surveyor auroral-type electron distributions [Brain et al., 2006a] and the electron distribution measured by ASPERA-3 on board Mars Express during SPICAM UVS observation. We also discussed different shapes of the auroral region, namely, a very elongated auroral region or an ideally spherical aurora region. Sources of uncertainty, like the neutral atmosphere and the cross sections for excitation of the observed emissions, have been also tested.

[23] The two clearly identified emissions during this auroral observation and in particular the ratio of their intensities, set a strong constraint on the parent electron energy distribution. An energy distribution peaking at low energy (below few tens of $\mathrm{eV}$ ) provides the best agreement with the observation. Interestingly, the emissions of atomic oxygen at 130.4 and $135.6 \mathrm{~nm}$ on the Venus nightside have also been suggested to be produced by such low-energy electron distributions [Fox and Stewart, 1991]. As a consequence, our best estimate for the electron population that may have produced the observation of SPICAM UVS is an intense flux of low-energy electrons, as an example, $\sim 10$ times more intense flux than the MGS magnetosheath typical electron distribution [Mitchell et al., 2001] or the flux observed by ASPERA-3 during SPICAM UVS observation corrected by a spacecraft potential smaller than $-10 \mathrm{~V}$ would be required. The auroral-type electron distributions observed by MGS [Brain et al., 2006a] and Mars Express [Lundin et al., 2006a, 2006b] would have produced a different distribution of the emissions than observed during this first aurora observation.

[24] Acknowledgments. F.L. thanks T. Slanger for helpful discussions which motivated this new analysis of SPICAM UVS observation.

[25] Wolfgang Baumjohann thanks Jane Fox and another reviewer for their assistance in evaluating this paper.

\section{References}

Acuňa, M. H., et al. (2001), Magnetic field of Mars: Summary of results from the aerobraking and mapping orbits, J. Geophys. Res., 106, 23,40323,417 .

Barabash, S., R. Lundin, H. Andersson, and The ASPERA-3 Team (2004), The analyzer of space plasmas and energetic atoms (ASPERA-3) for the Mars Express mission. In: Mars Express-The scientific payload, Eur. Space Agency, Spec. Publ., 1240, 121-139.

Bertaux, J. L., et al. (2000), The study of the Martian atmosphere from top to bottom with SPICAM light on Mars Express, Planet. Space Sci., 48, $1303-1320$

Bertaux, J.-L., F. Leblanc, O. Witasse, E. Quémerais, J. Lilensten, A. S. Stern, B. Sandel, and O. Korablev (2005a), Discovery of aurora on Mars, Nature, 435, 790-794.

Bertaux, J.-L., et al. (2005b), First observation of nightglow in the upper atmosphere of Mars: The NO bands in UV and implications for atmospheric transport, Science, 307, 566-569.

Bertaux, J. L., et al. (2006), SPICAM on Mars Express: Observing modes and overview of UV Spectrometer data and scientific results, J. Geophys. Res., doi:10.1029/2006JE002690, in press.

Blelly, P.-L., J. Lilensten, A. Robineau, J. Fontanari, and D. Alcayde (1996), Calibration of a numerical ionospheric model with EISCAT observations, Ann. Geophys., 14, 1375-1390. 
Brain, D. A., J. S. Halekas, L. M. Peticolas, R. P. Lin, J. G. Luhmann, D. L. Mitchell, G. T. Delory, S. W. Bougher, M. H. Acuňa, and H. Rème (2006a), On the origin of aurorae on Mars, Geophys. Res. Lett., 33, L01201, doi:10.1029/2005GL024782.

Brain, D. A., D. L. Mitchell, and J. S. Halekas (2006b), The magnetic field draping direction at Mars from April 1999 through August 2004, Icarus, 182, 464-473.

Conway, R. R. (1981), Spectroscopy of the Cameron bands in the Mars airglow, J. Geophys. Res., 86, 4767-4775.

Erdman, P. W., and E. C. Zipf (1983), Electron impact excitation of the Cameron system $\left(\mathrm{a}^{3} \pi \rightarrow \mathrm{X}^{1} \Sigma\right)$ of CO, Planet. Space Sci., 31, 317-321.

Fox, J. L. (1992), Airglow and aurora in the atmosphere of Venus and Mars, in Venus and Mars: Atmospheres, Ionospheres, and Solar Wind Interactions, Geophys. Monogr. Ser., vol. 66, edited by J. G. Luhmann, M. Tatrallyay, and R. O. Pepin, p. 191, AGU, Washington, D. C.

Fox, J. L., and A. Dalgarno (1979a), Electron energy deposition in carbon dioxide, Planet. Space Sci., 27, 491-502.

Fox, J. L., and A. Dalgarno (1979b), Ionization, luminosity, and heating of the upper atmosphere of Mars, J. Geophys. Res., 84, 7315-7333.

Fox, J. L., and A. I. F. Stewart (1991), The Venus ultraviolet aurora: A soft electron source, J. Geophys. Res., 96, 9821-9828.

Frahm, R. A., et al. (2006), Carbon dioxide photoelectron peaks at Mars, Icarus, $182,371-382$.

Haider, S. A., J. Kim, A. F. Nagy, C. N. Keller, M. I. Verigin, K. I Gringauz, N. M. Shutte, K. Szego, and P. Kiraly (1992), Calculated ionization rates, ion densities, and airglow emission rates due to precipitating electrons in the nightside ionosphere of Mars, J. Geophys. Res., 97, $10,637-10,641$

Haider, S. A., S. P. Seth, E. Kallio, and K. I. Oyama (2002), Solar EUV and electron-proton-hydrogen atom-produced ionosphere on Mars: Comparative studies of particle fluxes and ion production rates due to different processes, Icarus, 159, 18-30.

Leblanc, F., J. Y. Chaufray, J. Lilensten, O. Witasse, and J.-L. Bertaux (2006), Martian dayglow as seen by the SPICAM 23 UV spectrograph on Mars Express, J. Geophys. Res., 111, E09511, doi:10.1029/2005JE002664

Liehmon, M. W., et al. (2006), Numerical interpretation of high-altitude photoelectron observations, Icarus, 182, 383-395.

Lilensten, J., and P. L. Blelly (2002), The TEC and F2 parameters as tracers of the ionosphere and thermosphere, J. Atmos. Sol. Terr. Phys., 64, 775793 .
Lundin, R., et al. (2006a), Ionospheric plasma acceleration at Mars: ASPERA-3 results, Icarus, 182, 308-319.

Lundin, R., et al. (2006b), Plasma acceleration above Martian magnetic anomalies, Science, 311, 980-983.

Mantas, G. P., and W. B. Hanson (1979), Photoelectron fluxes in the Martian ionosphere, J. Geophys. Res., 84, 369-385.

Mitchell, D. L., R. P. Lin, C. Mazelle, H. Réme, P. A. Cloutier, J. E. P. Connerney, M. H. Acuňa, and N. F. Ness (2001), Probing Mars' crustal magnetic field and ionosphere with the MGS electron reflectometer, J. Geophys. Res., 106, 23,419-23,427.

Morel, L., O. Witasse, R. Warnant, J. C. Cerisier, P. L. Blelly, and J. Lilensten (2004), Diagnostic of the dayside ionosphere of Mars using the Total Electron Content measurement by the NEIGE/Netlander experiment, Planet. Space Sci., 52(7), 603-611.

Nier, A. O., and M. B. McElroy (1977), Composition and structure of Mars' upper atmosphere: Results from the neutral mass spectrometers on Viking 1 and 2, J. Geophys. Res., 82, 4341-4349.

Tian, C., and C. R. Vidal (1998), Electron impact dissociative ionization of $\mathrm{CO}_{2}$ : Measurements with a focusing time-of-flight mass spectrometer, J. Phys. Chem., 108, 927-936.

Verigin, M. I., K. I. Gringauz, N. M. Shutte, S. A. Haider, K. Szego, P. Kiraly, A. F. Nagy, and T. I. Gombosi (1991), On the possible source of the ionization in the nighttime Martial ionosphere: 1 . PHOBOS 2 harp electron spectrometer measurements, J. Geophys. Res., 96, 19,307-19,313.

Witasse, O., et al. (2002), Prediction of a $\mathrm{CO}_{2}^{2+}$ layer in the atmosphere of Mars, Geophys. Res. Lett., 29(8), 1263, doi:10.1029/2002GL014781.

J. L. Bertaux and F. Leblanc, Service d'Aeronomie du CNRS, Reduit de Verrieres BP 3, Route des Gatines, F-91371 Verrieres-Le-Buisson, France. (francois.leblanc@aerov.jussieu.fr)

P.-L. Blelly, Laboratoire de Physique et Chimie de l'Environnement, F-45071 Orléans Cedex 2, France.

D. Brain and J. S. Halekas, Space Sciences Laboratory, University of California, Berkeley, CA 94720, USA

R. A. Frahm and J. Winningham, Southwest Research Institute, 6220 Culebra Road, P.O. Box 28510, San Antonio, TX 78228-0510, USA.

J. Lilensten, Laboratoire de Planétologie de Grenoble, BP 53, F-38041 Grenoble, France.

O. Witasse, Research and Scientific Support Department of ESA-ESTEC, P.O. Box 299, NL-2000 AG Noordwijk, Netherlands. 